\title{
Proceedings of the 125th Meeting of the Society of British Neurological Surgeons, University of Dundee, 7-9 September 1994
}

FAMILIAL FRONTONASAL DERMOID CYSTS WTH CALLOSAL AGENESIS

I Jacobson, MA Jeeves, W Mcnab. Royal Infirmary, Dundee and the University of $\mathrm{St}$ Andrews, UK

Objective-To report a unique family in which three generations have presented with frontonasal dermoids, epilepsy, and significant psychometric and interhemisphere transfer abnormalities.

Design-Over a 30 year period eight members of a family have been clinically and radiologically studied with extensive psychometric and neurophysiological confirmation. Five have been operated on.

Results-The syndrome described has a poor long term outcome particularly in respect of seizure control and the development of progressive dementia. Although familial nasal dermoid cysts and also familial syndromes of callosal agenesis have been documented to date, ${ }^{12}$ no similar family has ever been recorded. Also, this syndrome seems to be progressive with increasing long term disability. This is also different from other callosal syndromes. A combined single stage frontonasal approach is recommended for surgical management of dermoid cysts.

1 Baarsma EA. The median nasal sinus and dermoid cyst. Archiv Otorhinolaryng 1980;226:

2 Aderman E. Agenesis of the corpus callosum. In: Vinken PJ, Bruyn GW, eds. Handbook for clinical neurology. Amsterdam: North Holland 1981;1:6-9.

AVOIDABLE RISK FACTORS AND POSTOPERATIVE HAEMATOMA

JD Palmer, OC Sparrow, F Iannotti. Wessex Neurological Centre, Southampton, UK

This study has examined the surgical practice at the Wessex Neurological Centre over a five year period from 1989 to 1993 to determine the incidence of postoperative haematoma $(\mathrm{POH})$ and identify risk factors for a perioperative bleeding disorder. The study included only those POHs (at any site) that followed, and were related to, a neurosurgical operation and were surgically evacuated. The study was prospective for the year 1993 and retrospective for the preceding years.

Over the five years 6668 operations were performed and $71 \mathrm{POHs}$ were surgically evacuated giving an overall rate of $1.06 \%$ of operations. The records were available on 69 cases. The most frequent diagnosis leading to POHs was meningioma surgery with a rate of $6 \cdot 16 \%$ of cases $(13 / 211)$, followed by craniotomy for trauma $3.65 \%(7 / 192)$, aneurysm surgery $2.57 \%(11 / 428)$, and intrinsic supratentorial tumours $2 \cdot 22 \%(10 / 451)$.

POHs were intracerebral (ICH) in $43 \%$, subdural in $5 \%$, extradural (EDH) in $33 \%$, mixed in $8 \%$, and wound in $11 \%$ of cases. The mortality from $\mathrm{POH}$ was $32 \%(37 \%$ for ICH, $12 \%$ for EDH). Risk factors for a perioperative bleeding disorder were present in $2 / 3$ of the patients. Administration of antiplatelet agents (aspirin, NSAIDs) was the most common risk factor, identified in 30 of the 69 patients. At least $75 \%$ of identified risk factors could have been avoided or corrected before surgery.

A RANDOMISED DOUBLE BLIND STUDY OF SODIUM VALPROATE FOR THE PREVENTION OF SEIZURES IN NEUROSURGICAL PATIENTS JP Holland, SR Stapleton, AJ Moore, HT Marsh, D Uttley, BA Bell. Atkinson Morley's Hospital, London, UK

Objective-Anticonvulsant drugs are commonly prescribed to prevent seizures that may follow head trauma or craniotomy despite no evidence from prospective studies of a truly prophylactic benefit. A randomised double blind study of sodium valproate has been performed to assess its efficacy in the prevention of postoperative seizures.

Design-A total of 301 patients were randomly assigned to treatment with sodium valproate $(n=152)$ or placebo $(n=149)$ for one year in a double-blind trial. An intravenous loading dose was given in the anaesthetic room before craniotomy or within 24 hours of head injury. Patients were stratified prospectively into high or low risk categories in the head injury and craniotomy groups. Serum concentrations of valproate were maintained in the therapeutic range $40-80 \mu \mathrm{g} / 1$ for one year and follow up was continued for two years from randomisation.

Results-The overall incidence of seizures was similar in both arms of the study, $21 \%$ for placebo and $18 \%$ for active treatment, but there was a significant reduction in the incidence of seizures in the low risk craniotomy group (non-space occupying lesions): $11 \%$ v $24 \% \quad(\mathrm{p}<0.01$ at six months, $\mathrm{p}<0.05$ at two years).

Conclusions-Sodium valproate given for 12 months exerts a prophylactic anticonvulsant effect in those patients undergoing a craniotomy for a non-space occupying lesion by significantly reducing the incidence of seizures up to two years from randomisation.

USE OF GUIDELINES FOR HEAD INJURY MANAGEMENT IN SCOTLAND

DL Oluoch-Olunya, AD Braes, GM Teasdale. Institute of Neurological Sciences, Glasgow and Scottish Health Management Efficiency Group, UK

Objectives-To discover current approaches to management of head injuries in Scotland, how this relates to published guidelines, and how well these are known.
Design-Questionnaires were sent on prehospital and hospital management to hospitals with accident and emergency departments in Scotland.

Subjects-Fifty one hospitals (teaching $\mathrm{n}=10$, district general $\mathrm{n}=16$, rural $/$ remote $n=25)$ in all the 13 Scottish boards were surveyed between April 1993 and February 1994.

Outcome measures-Facilities available and type of guidelines used.

Results-Ninety seven per cent of the hospitals had a local 24 hour skull $x$ ray service but only $24 \%$ had a local CT service. Teaching and district general hospitals used guidelines, but in only $39 \%$ were these derived from the Harrogate Seminar ${ }^{1}$ and the guidelines of $1984 .^{2}$ Thirty six per cent of the remote/rural hospitals did not use any type of guidelines.

Conclusion-The guidelines published a decade ago need to be revised to take account of changes in CT availability and clinical practices. There is a need to target the remote/rural hospitals to use head injury management guidelines.

1 Lewis AF, ed. The management of acute head injury. Harrogate Seminar Report 8. London: DHSS, 1983.

2 Guidelines for the initial management after head injury in adults. $B M \mathcal{F}$ 1984;288:983-5.

MONITORING OF INTRACRANIAL OXY AND DEOXYHAEMOGLOBIN LEVELS IN HEAD INJURED PATIENTS USING NEAR INFRARED SPECTROSCOPY: ARE CALCULATIONS OF CEREBRAL HAEMOGLOBIN SATURATION VALID?

PJ Kirkpatrick, P Smielewski, M Czosnyka, JD Pickard. Addenbrookes Hospital, Cambridge, UK

Near infrared spectroscopy (NIRSHamamatsu 1000) and jugular venous oximetry (JVO-Oximetrix 3) have been employed in six ventilated, severely head injured patients to monitor potential changes in cerebral oxygenation. In all patients, NIRS demonstrated transient reductions in oxygenated haemoglobin $\left(\mathrm{HbO}_{2}\right)$ levels during waves of raised intracranial pressure (ICP) that were closely correlated with transcranial Doppler middle cerebral artery flow velocities (FV) and cortical perfusion (laser Doppler flowmetryLDF). Although about $15 \%$ of these events were correlated with JVO changes, most episodes of potential desaturation during raised ICP were not registered by JVO.

During stable states of ICP, FV, LDF, JVO, and NIRS, spontaneous variations in the $\mathrm{Hb}$ and $\mathrm{HbO}_{2}$ signals were found. On calculating the ratio $\left[\Delta \mathrm{HbO}_{2}\right] /\left[\Delta\left(\mathrm{HbO}_{2}+\right.\right.$ $\mathrm{Hb})$ ] derived during 10 minute epochs, a theoretical value for cerebral haemoglobin saturation (HbSat) can be calculated. Comparison with static JVO measurements 
calibrated with co-oximetry indicated a reasonable correlation $\left(r^{2}=0 \cdot 68\right)$. The NIRS derived HbSat, however, assumes a constant saturation during the sampling epoch which is affected by changes in cerebral blood volume. These conditions may not be satisfied during dynamic changes in ICP and cerebral blood flow. Indeed, NIRS derived calculations of $\mathrm{HbSat}$ during ICP waves showed poor correlation with JVO, and occasionally gave results which were not sensible (for example, HbSat $>100 \%$ ).

It is concluded that whereas NIRS derived $\mathrm{HbO}_{2}$ signal changes are sensitive to cerebral haemodynamic changes that predict focal ischaemia, algorithms that calculate continuous and dynamic changes in $\mathrm{HbSat}$ from rapid variations in $\mathbf{H b O}_{2}$ and $\mathrm{Hb}$ signals should not be regarded as reliable.

EFFECT OF EXTRACEREBRAL TISSUE ON NONINVASIVE MEASUREMENT OF CEREBRAL BLOOD FLOW BY NEAR INFRARED SPECTROSCOPY

H Owen-Reece, CE Elwell, $M$ Smith, DT Delpy, JS Wyatt, WFJ Harkness. National

Hospital for Neurology and Neurosurgery and University College, London, UK

Near infrared spectroscopy (NIRS) is a technique with potential for quantifying cerebral blood flow (CBF) non-invasively. The theoretical and practical aspects ${ }^{1}$ have previously been described and CBF has been measured in conscious and anaesthetised subjects. ${ }^{2}$ Median CBF during anaesthesia was $25 \mathrm{ml} .100 \mathrm{~g}^{-1} \cdot \mathrm{min}^{-1}$, which is lower than typically obtained by alternative methods. It was predicted that this effect was due to extracerebral tissue and would not be seen in measurements made on the dura. The purpose of this study was to obtain measurements of CBF by positioning the optical fibres on the dura in patients undergoing craniotomy.

Six patients undergoing excision of a temporal lobe epileptic focus were anaesthetised with a standard technique. Before surgery CBF was measured as described, ${ }^{1}$ after craniotomy, CBF measurements were repeated on the dural surface. In each patient arterial $\mathrm{pCO}_{2}$ and blood pressure were standardised for the two measurements.

Mean CBF measured on the scalp was 21 (8) and on the dura 67 (23) $\mathrm{ml} .100 \mathrm{~g}^{-1} \cdot \mathrm{min}^{-1}$. The difference was highly significant $(p<0.001)$. The most likely explanation is light scattering by extracerebral tissue. These data support the theoretical validity of CBF measurement by NIRS.

1 Elwell CE, et al. Measurement of cerebral blood flow in adult humans using near infrared spectroscopy-methodolooy and possible errors. Adv Exp Med Biol 1992. 317:235-45.

2 Owen-Reece $\mathrm{H}$, et al. Use of near infrared spectroscopy to measure cerebral blood flow spectroscopy to measure cerebral blood flow f Anaesth 1994 (in press).

EFFECT OF CLOWARD'S ANTERIOR CERVICAL FUSION ON INTERVERTEBRAL FORAMINAL SIZE

RD Strachan, CGH West. Hope Hospital, Salford, UK

Objective-The presumed effect of Cloward's cervical fusion on intervertebral foraminal topography is largely anecdotal.
Many surgeons believe that restoration of disc height and intervertebral foraminal dimensions are important for a successful surgical outcome. Few efforts have been made, however, to quantify what happens to the configuration of the intervertebral foramen after insertion of a cylindrical graft. This prospective study considers this question.

Design-Using standardised preoperative and postoperative oblique cervical spine radiographs, image magnification, cross sectional planimetry, and computer assisted analysis, the effects of Cloward's anterior cervical fusion on intervertebral foraminal dimensions have been quantified.

Results-A study of the first 12 patients confirmed that after fusion, mean cross sectional area increased from $0.66(0.03) \mathrm{cm}^{2}$ to $0.75(0.05) \mathrm{cm}^{2}$ or by an average $13.5 \%$. This increase in area was significant when compared with control non-operative values $(p<0.05)$. The maximum transverse foraminal dimension also significantly increased from $12.6(0.31) \mathrm{mm}$ to 13.6 $(0.38) \mathrm{mm}$ after surgery $(p<0.05)$. This change was highly significant when compared with the control non-operative values (p $<0.005$ ).

Conclusion-Cloward's discectomy and fusion does increase the intervertebral foraminal dimensions.

DOES THE USE OF BOP OCTA PROVIDE A SUITABLE ALTERNATIVE TO ILIAC CREST AUTOLOGOUS BONE GRAFT IN ANTERIOR CERVICAL DISCECTOMY AND FUSION

T Sattar, A Penrose-Stevens, JRS Leggate. North Manchester General Hospital, Crumpsall, UK

In anterior cervical discectomy and fusion, whether by the technique of Cloward or of Smith-Robinson, the major cause of morbidity and prolonged stay in hospital is related to the bone graft donor site. Substitutes for autologous bone have been tried but have failed due to mechanical collapse, non-union, or graft rejection. In a series of 36 patients ( $61 \%$ women, $39 \%$ men) the bone graft was replaced by an acrylic polymer implant, BOP (octa). The mean age of the group was 46.5 years and $29(81 \%)$ underwent a single level fusion and seven $(19 \%)$ a two level fusion. The commonest level involved was C5/6 (56\%) and then C6/7 and C4/5. Preoperative MRI was obtained in $25(70 \%)$ and postoperative MRI or CT were performed in nine patients $(27 \%)$. The median inpatient stay was $5 \cdot 5$ days, and mean follow up was 13 months (range 6-24). Anterior prominence has been noted on MRI in one patient, but to date no graft rejection, infection, or collapse has been found. It seems that BOP (octa) may be a suitable alternative to iliac crest bone. This pilot data is the basis for a prospective trial of BOP (octa) in anterior cervical spinal surgery.

LATERAL BRANCH OF THE POSTERIOR PRIMARY RAMUS-KEY FOR THE POSTEROLATERAL APPROACH TO EXTREME LATERAL LUMBAR DISC HERNIATIONS M O'Brien, D Peterson, HA Crockard. The National Hospital for Neurology and Neurosurgery, London, UK

Introduction-Extreme lateral lumbar disc herniations (ELLDH) are reliably detected on MRI and CT. Access remains problematic with conventional posterior approaches.
Objective-To identify constant anatomical landmarks which will facilitate access to these disc herniations via an approach that does not require bone resection.

Design-(a) Anatomical dissections: Bilateral lumbar spine dissections were performed on 10 cadavers. The lateral branch of the posterior primary ramus (LBPPR) was identified and its relation to adjacent vertebral structures was analysed. (b) Operative procedures: A longitudinal skin incision 8-10 cm from the midline was followed by an oblique posterolateral muscle splitting approach. Microsurgical techniques were used for nerve root decompression.

Patients-Nine men and one woman were explored for 10 ELLDHs identified at L1-2 in one patient, L2-3 in one patient, L3-4 in three patients, $L 4-5$ in three patients, and at $\mathrm{L} 5-\mathrm{S} 1$ in two patients.

Outcome measures-Postoperative relief of nerve root compression symptoms.

Results-(a) Anatomical dissections: Accompanied by the terminal branch of the segmental artery, the LBPPR was consistently found medially between the longissimus and the intertransverse muscle overlying the transverse process of the adjacent caudal vertebra. Laterally it entered the iliocostalis. (b) Operative Procedures: Blunt dissection with the index finger consistently identified the LBPPR as a taut band in the iliocostalis. This was followed to the intervertebral foramen. Decompression was achieved without bone resection. Operative time ranged from 45-95 minutes. All patients obtained relief of clinical symptoms.

Conclusions-Identification of the LBPPR during the posterolateral approach facilitates rapid localisation and safe removal of ELIDHs without bone resection.

THE PATHOGENESIS OF PERIDURAL FIBROSIS-A MACROSCOPIC, MICROSCOPIC, AND IN SITU HYBRIDISATION STUDY

KM Morris, AJ Freemont, JA Hoyland, MIV Jayson. Department of Neurosurgery and Rheumatology, Hope Hospital, Manchester and Department of Pathology, University of Manchester, UK

Objective-To determine the time course and pathological processes leading to the formation of peridural fibrosis after disca surgery in a rabbit model and to re-evaluate the proposed role of peridural haematoma. ${ }^{1}$

Design-Macroscopic and microscopic observational study of the formation of peridural scar including evaluation of temporal and spatial changes in mRNA expression for types I, II, and III collagen and fibroblast collagenase by in situ hybridisation.

Subjects-Twenty rabbits with 40 macroscopically and 40 microscopically studied surgical sites were examined at two and fou days and $1,2,3,6,9,12,15$, and 18 weeks after trans-spinal discal surgery.

Outcome measures-Four point graded macroscopic dissection and observation of matrix formation during peridural repair. Microscopic observation of the formation of peridural fibrosis and correlation with changes in matrix protein mRNA expression.

Results-Formation of clinically significant scarring was delayed until three weeks postsurgery. Blood clot was cleared from the peridural space by one week and replaced by an "inflammatory exudative" 
provisional matrix which together with biomechanical factors maintained contact between injured dural and canal surfaces.

Fibroblast proliferation occurred from all injured surfaces except the dura. Type III collagen mRNA was expressed at the dura canal interface for three weeks, and type I mRNA for six weeks after surgery. Collagenase mRNA was expressed for six weeks after surgery. Type II collagen mRNA was not expressed at the dural interface.

Prolonged matrix protein mRNA expression occurred in deep structures in association with remodelling or secondary degeneration.

Conclusions-Peridural wound healing was similar to repair in other tissues. Mechanical apposition, formation of an exudative matrix, and prolonged production of matrix proteins are likely to be at least as important as peridural haematoma in the formation of peridural fibrosis. The potential for matrix deposition persists for at least six weeks after surgery.

1 LaRocca H, Macnab I. The laminectomy membrane. Study of its evolution, characteristics, effects and prophylaxis in dogs. f Bone foint Surg Br 1974;56B:545-50.

2 Songer MI, Ghosh L, Spencer DL. Effects of sodium hyaluronidate on peridural fibrosis after lumbar discectomy. Spine 1990; 15:550-4.

DOES SURGERY ON THE RHEUMATOID CERVICAL SPINE BENEFIT THE BED BOUND, NON-AMBULATORY (RANAWAT 3B) MYELOPATH? 3B OR NOT 3B ? ATH Casey, HA Crockard. The National Hospital, London, UK

Objectives-To determine the efficacy of cervical spine surgery on Ranawat class $3 b$ patients, and to identify by statistical analysis the prognostic factors determining surgical success.

Design-A prospective study of rheumatoid patients undergoing surgery for myelopathy secondary to atlantoaxial subluxation during a 10 year period 1983-93.

Patients-There were 256 patients treated for cervical spine involvement by the rheumatoid process of which 55 from Ranawat class $3 \mathrm{~b}$ underwent surgery.

Outcome measures-Neurological outcome was assessed using the Ranawat classification (1-3b), and functional outcome using the Health Activity Questionnaire (HAQ) disability index. Statistical analysis was performed by univariate analysis, multiple logistic regression, and survival analysis, with significance accepted at the $5 \%$ level.

Results-There were 55 class $3 \mathrm{~b}$ patients (mean age $65 ; 44$ women, 11 men) who underwent surgery either by a combined anterior transoral and posterior approach (37) or a posterior approach alone (18). The mean duration of hospital stay was 26 days of which 5.3 days were spent in ITU. Postoperative morbidity was recorded in $\mathbf{2 7}$ patients $(49 \%)$ and the 30 day mortality was $12 \cdot 7 \%$. During follow up a further 24 patients have died $(47 \%)$ with a mean time to death of only 14.4 months. Significant neurological or functional improvement was seen in 14 patients $(24.5 \%)$. Adverse prognostic factors included increasing age, level of pre-operative disability, spinal canal diameter, and spinal cord area.

Conclusions-Surgery on Ranawat class 3b carries a high morbidity and mortality with only modest improvement in neurolog- ical or functional outcome. Surgical referral patterns need to be improved and it is hoped that prompt surgery before the development of irreversible cord damage and atrophy may yield more favourable results.

COMPLEX DISORDERS OF THE PAEDIATRIC CRANIOCERVICAL JUNCTION

HA Crockard, ATH Casey, WFJ Harkness, RD Hayward. The National Hospital and the Hospital for Sick Children, London, UK

Osseous anomalies affecting the paediatric craniocervical junction malformation are rare making it difficult for any one surgeon to accrue significant experience in this area. To redress this deficiency a paediatric craniocervical clinic has been created which combines the experiences of the departments of surgical neurology at the Nationa Hospital and those at Great Ormond Street. The purpose of this presentation is to present the first year's experience.

Material and methods-Eleven patients (four male; seven female) mean age 5.6 years, have been treated to date. Their preoperative diagnoses included spondyloephyseal dysplasia (four), cervical dysplasia (one), otopalatal digital syndrome (one), achondroplasia (one), Morquio's syndrome (one), two cases of atlantoaxial rotary dislocation, and congenital atlantoaxial subluxation (one). Abnormalities encountered in the congenital group included os odontoideum, atlantoaxial subluxation, anterior and posterior spina bifida, and seg mentation defects. Preoperative evaluation by other paediatric specialists has been particularly informative. Understanding of the pathomechanics of these complex problems and surgical planning is assisted by CT (both two and three dimensional reconstructions), MRI, and angiography in selected cases.

Surgical technique-The surgical approach was tailored to the individual case with three approaches being used (transoral, far lateral, and posterior). A halo device was applied preoperatively when instability was anticipated. For stabilisation and fusion we have employed a technique that uses autologous calvarium harvested from the parietoccipital region. It is secured by the titanium sof' wires $^{(\mathrm{TM})}$, which facilitates postoperative imaging. The resultant bony defect is repaired by using split thickness calvarium.

The management of these complex cases and their complications shows the advantages of a combined speciality clinic in units with a full array of paediatric services.

DOES THREE DIMENSIONAL MAGNETIC RESONANCE ANGIOGRAPHY SUBSTITUTE FOR CONVENTIONAL ANGIOGRAPHY IN SURGICAL MANAGEMENT OF RUPTURED CEREBRAL ANEURYSM?

DA Jellinek, D Wilcock, JL Firth, T Jaspan, I Holland, B Worthington. Departments of Neurosurgery and Radiology, University Hospital, Nottingham, UK

Objective-To determine -if magnetic resonance angiography (MRA) is an acceptable substitute for conventional angiography in surgical management of subarachnoid haemorrhage (SAH)

Design-Comparison of the sensitivity and specificity of MRA with conventional angiography in the detection and surgical characterisation of cerebral aneurysm in patients with a proved diagnosis of recent SAH.

Patients-Twenty patients with proved diagnosis of SAH had both MRA and conventional angiography.

Method-A three dimensional "time of flight" MR angiogram sequence was performed before surgery to image the circle of Willis, the cavernous carotid arteries, the M1 segments, and the posterior circulation to the origin of the PICAs. The examining radiologist (blind to the results of conventional angiography) recorded whether or not he thought an aneurysm was present. When an aneurysm was found on MRA the images were reviewed neurosurgically to establish if sufficient information was available for surgery; MRA images were also reviewed in the light of operative findings and compared with the conventional angiogram

Results-MRA was a sensitive and specific test for the diagnosis of cerebral aneurysm. The MRA also provided information not available from the conventional angiogram about the distal (clotted) aneurysm sac. In the presence of a large perianeurysmal haematoma, however, surgical characterisation of the aneurysm was still difficult.

Conclusion-MRA has a valid role in the management of aneurysmal subarachnoid haemorrhage.

MAGNETIC RESONANCE ANGIOGRAPHY IN THE MANAGEMENT OF ANEURYSMAL

SUBARACHNOID HAEMORRHAGE: A STUDY OF 51 CASES

S Sankhla, WJ Gunawardena, CMA Coutinho, AJ Keogh. Royal Preston Hospital, Preston, UK

Objective-To assess the usefulness of magnetic resonance angiography (MRA) in the management of subarachnoid haemorrhage (SAH) from a suspected berry aneurysm with the object of early surgical obliteration of any aneurysms found.

Method-Review of 51 patients who had sustained a SAH and were investigated by MRA. Fifty aneurysms at a variety of site were identified in 43 patients, with norma results in eight. MRA was performed (Siemens 1.5 T Magnetom) using a three dimensional time of flight sequence ${ }^{2}$ as well as standard MRI. Intra-arterial digital subtraction angiography (IA DSA) was also performed at some stage.

Results-(a) Group 1: 38 patients (75\%) were classified as having satisfactory and accurate MRA results (findings (32 with aneurysms and six without) were confirmed at surgery and/or on IA DSA). On the basis of the MRA results alone 20 patients (23 aneurysms) underwent surgery before IA DSA. (b) Group 2: Thirteen patients (25\%) were classified as having unsatisfactory MRA results, nine had movement artefacts three false negatives $(6 \%)$, and one false positive (2\%).

Conclusion-The safe and non-invasive investigation of MRA gives satisfactory information in a high percentage of patients sustaining an SAH, 38/51 (74.5\%), allowing early surgery without recourse to IA DSA in most $20 / 32(62.5 \%)$

Gouliamos A, Gotsis E, Vlahos L, et al. Magnetic resonance angiography compared to intra-arterial digital subtraction angiography in patients with subarachnoid haemorrhage. Neuroradiology 1992;35:46-9. 
2 Ross JS, Mararyk TJ, Modic MT, et al. Intracranial aneurysms: evaluation by $\mathbf{M R}$ angiography. Am $\mathcal{F}$ Neuroradiol 1990; 11:449-56.

A PROSPECTIVE RANDOMISED TRIAL OF EFFICACY OF TIRILAZAD MESYLATE IN PATIENTS WITH SUBARACHNOID HAEMORRHAGE

AD Mendelow. European/Australian Tirilazad SAH Study Group

Tirilazad mesylate (U-74, 006F) is a synthetic non-glucocorticoid steroid that inhibits iron catalysed lipid peroxidation. A prospective randomised placebo controlled clinical trial of three doses $(0 \cdot 6,2 \cdot 0$, and 6.0 $\mathrm{mg} / \mathrm{kg} /$ day) given at six-hourly intervals for up to 10 days was conducted in patients with CT or LP confirmed subarachnoid haemorrhage and angiographically confirmed aneurysms. A total of 1015 patients from 41 centres was randomised and received at least one dose of treatment. All patients received nimodipine. End points included the functional outcome on the Glasgow outcome scale (GOS) at three months, incidence and severity of angiographic and symptomatic vasospasm, and CT infarction at 14 days and three months.

There was no significant difference in the primary end point of favourable outcome (good recovery/moderate disability) compared with unfavourable outcome (severe disability/vegetative/dead) between placebo and the three doses given, although at the highest dose favourable outcomes occurred in $72 \%$ of treated patients compared with $67 \%$ of controls. There was a significant reduction in mortality at the highest dose $(6.0 \mathrm{mg} / \mathrm{kg} / \mathrm{day})$ compared with placebo $(12 \% v 21 \%)$ and a significant increase in the number of patients who made a good recovery ( $64 \% v 53 \%)$. There were also no significant differences in the proportion of treated and untreated patients with CT infarction (nor in the volume of infarction) at three months.

CHANGES IN MICROVASCULAR PERMEABILITY IN A RODENT MODEL OF FOCAL CORTICAL INJURY USING ${ }^{14} \mathrm{C}$ AIB AUTORADIOGRAPHY: THE EFFECT OF TIRILAZAD MESYLATE

P Mathew, DI Graham, R Bullock, J McCulloch, GM Teasdale. Institute of Neurological Sciences, Glasgow, UK

Objective-To determine the changes in microvascular permeability with time in a new rodent model of focal cortical injury, and to assess the effects of Tirilazad.

Design-Forty adult Sprague-Dawley rats were studied. Focal cortical contusions were produced by applying a localised suction impact. ${ }^{1}$ Animals were randomly allocated to sham operated $(n=5)$, lesion $(n=5)$, and Tirilazad tested $(n=5)$ groups for each survival time of $\mathbf{3 0}$ minutes, four hours, and 24 hours. Animals receiving Tirilazad were given $10 \mathrm{mg} / \mathrm{kg}$ intravenously, five minutes after injury.

End points-Measurement of cerebral microvascular permeability by ${ }^{14} \mathrm{C}$ AIB quantitative autoradiography, ${ }^{2}$ and volumetric assessment of the autoradiographic lesion.

Results-Compared with sham operated animals there was a significant increase in perilesional microvascular permeability at all time points studied; this increase was most pronounced at four hours (mean $k$ $(S E M)=21.9(0.6 / 1000) \mathrm{min})$, and coincided with the greatest volume of the lesion (mean volume $\left.(\mathrm{SE})=15.48(0 \cdot 7) \mathrm{mm}^{3}\right)$. In tirilazad treated animals at four hours there was a significant reduction in microvascular permeability $(k=3.1(0.5), p<0.001)$ and lesion volume $\left(4.86(0.7) \mathrm{mm}^{3}, \mathrm{p}<0.01\right)$.

Conclusions-In this model a delayed increase in microvascular permeability occurs which is significantly minimised by postinjury treatment with IV tirilazad.

1 Mathew P, Graham DI, Bullock R, et al. Focal brain injury: histological evidence of delayed inflammatory response in a new rodent model of focal contusion injury. Acto Neurochir (Wein) 1994;60(suppl 1):428-30. Blasberg RG, Patlak CS, Jehle JW, et al. An autoradiographic technique to measure the permeability of normal and abnormal brain capillaries. Neurology 1978;28:363.

INTRATHECAL CALCITONIN GENE-RELATED PEPTIDE REDUCES ISCHAEMIC BRAIN DAMAGE JP Holland, BA Bell. Atkinson Morley's Hospital, London, UK

Objective-Calcitonin gene-related peptide (CGRP) is an endogenous vasodilating neuropeptide which has a selective action on the cerebral arteries causing an increase in cerebral blood flow. ${ }^{1}$ High doses of intravenous CGRP are known to cause systemic vasodilatation resulting in an unwanted drop in blood pressure, ${ }^{2}$ and this study set out to test the efficacy of the peptide given intrathecally.

Design-A series of dose response experiments have defined an intrathecal bolus dose of CGRP of $225 \mathrm{ng} / \mathrm{kg}$ which increased cerebral blood flow by $28 \%$ in the anaesthetised rat but had no effects on systemic blood pressure. Using this dose the effects of pretreatment with intrathecal CGRP $(n=10)$ compared with normal saline $(n=10)$ were studied in a model of focal cerebral ischaemia.

Results-The treatment group maintained a higher cerebral blood flow (31 mv/100 $\mathrm{g} / \mathrm{min}$ compared with $15 \mathrm{ml} / 100 \mathrm{~g} / \mathrm{min}, \mathrm{p}<$ 0.01 ) and the concomitant volume of ischaemic brain injury was reduced by $57 \%$ (94 (18) compared with 204 (21) $\mathrm{mm}^{2} \mathrm{p}<$ $0.001)$. There was no systemic hypotension.

Conclusions-These findings show the potential benefits of intrathecal CGRP in focal cerebral ischaemia.

1 Edvinnson $\mathrm{L}$ et al. Calcitonin gene-related peptide and cerebral blood vessels; distribupeptide and cerebral blood vessels; distribuFlow Metab 1986;7:720-8.

2 European CGRP in subarachnoid haemorrhage study group. Effect of CGRP in patients with delayed post-operative cerebral haemorrhage. Lancet 1992;339:831-4.

POSSIBLE THERAPEUTIC ROLE FOR ENDOTHEI IN ANTAGONISTS

S Galbraith, T Patel, M McAuley, J McCulloch. Wellcome Surgical Institute and the Department of Neurosurgery, University of Glasgow, UK

Endothelins are implicated as a cause of neural damage after cerebral ischaemia. Their role in focal cerebral ischaemia and the therapeutic possibilities of their modification by the endothelin antagonist bosentan has been examined.

Materials and methods-In anaesthetised adult cats a craniectomy was made over the parietal cortex and the dura reflected. The middle cerebral artery was permanently occluded via a transorbital approach.
Arteriolar calibre was measured with an image splitter. Artificial CSF or drugs dissolved in CSF were injected into the perivascular subarachnoid space.

Results-Occlusion of the middle cerebra artery produced a mixture of vasoconstriction and vasodilatation. The perivascular microapplication of bosentan $(30 \mu \mathrm{M})$ resulted in an increase in arteriolar diameter of $20 \%$ ( $p<0.001 v \mathrm{CSF}$ ). The numerical increase was greater in vessels which were constricted after induction of ischaemia.

Conclusions-Endogenous endothelins constrict or impair the dilatation of cortical vessels in the territory of focal ischaemia and this can be attenuated by the endothelin antagonist bosentan. Endothelin antagonists may therefore have a therapeutic role following cerebral ischaemia.

RESECTION OF THE PATHOLOGICAL SUBSTRATE IN THE TEMPORAL NEOCORTEX JP Phillips, O Hardiman, M Farrell, H Staunton. Beaumont Hospital, Dublin, Ireland

Fifty patients underwent superficial temporal lobectomy for intractable temporal lobe epilepsy. Total cure rate was $52 \%$ and significant improvement was achieved in $\mathbf{8 8 \%}$. Cytoarchitectural changes in grey and white tissue were analysed under light microscopy. Neuronal dysgenesis was correlated with the duration of seizure disorder, age on onset, and other aetiological factors and with clinical outcome. Temporal lobes from 33 neurologically normal necropsy brains which were age and sex matched with patients were examined as controls Severe neuronal ectopia ( $>8$ neurons/2 $\mathrm{mm}^{2}$ white matter) was present in $42 \%$ of patients with epilepsy and in none of the controls. There was neuronal clustering in $28 \%$ of those with epilepsy and Chaslin's (subpial) gliosis in $38 \%$. Controls did not have these changes. The presence of severe neuronal ectopia and clustering was predictive of a favourable clinical outcome after surgery $(p<0.05)$. No correlation was found between microdysgenesis and other factors. These findings suggest that the presence of neuronal dysgenesis may be of significance in the clinical outcome after surgery, and that the abnormal tissue may be important as a morphological substrate for seizures in some patients. It is proposed that micro dysgenesis is one spectrum of cortical dysplasia and that resection of this pathological substrate is in effect a lesionectomy procedure.

ICTAL SPECT AS A LOCALISING INVESTIGATION IN EXTRATEMPORAL EPILEPSIES

R Duncan, J Patterson, K Lindsay, $\mathbf{R}$ Roberts, D Hadley. Institute of Neurological Sciences, Glasgow, UK

Ictal HMPAO SPECT has proved reliable as a localising investigation in mesial temporal lobe epilepsies, but focal epilepsies originating elsewhere in the brain have been little studied.

Ictal HMPAO SPECT images in 10 patients with focal epilepsies are presented where other localising data (seizure semiology, interictal and ictal EEG, MRI) have shown extratemporal origin, in eight patients in different areas of the fronta lobe, and in two patients at the temporooccipital junction. 
All 10 patients showed hyperperfusion at the site of the focus. Among the eight frontal lesions, two patients also showed activation of parts of the contralateral frontal lobe. Activation of the basal ganglia, thalamus, and/or cerebellum were seen in five patients, as well as hypoperfusion of the hemisphere contralateral to the focus in three patients, and ipsilateral in one. In the two temporo-occipital lesions, activation of the ipsilateral lateral temporal lobe was seen, involving the mesial temporal structures and basal ganglia to a lesser degree. Ictal hypoperfusion in these two patients was ipsilateral to the focus.

Ictal rCBF patterns may be complex in extratemporal seizures, but do seem to distinguish them from mesial temporal seizures and to provide useful localising information.

CORRELATION BETWEEN PRE-OPERATIVE MRI DIAGNOSIS AND HISTOLOGICAL FINDINGS IN PATIENTS UNDERGOING SURGERY FOR

INTRACTABLE EPILEPSY

J Allibone, N Alsanjari, DR Fish, J Duncan, H Cross, B Neville, WFJ Harkness. The National Hospital for Neurology and Neurosurgery and the Hospital for Sick Children, London, UK

Objective-To demonstrate the value of MRI, as part of a predominantly non-invasive protocol, in the preoperative assessment of patients undergoing surgery for medically intractable epilepsy.

Design-Comparison of the preoperative anatomical MRI diagnosis with the histological findings after surgery for medically intractable seizures.

Patients-The first 100 patients who were operated on by the senior surgical author as treatment for epilepsy.

Results-Seventy-four patients presented with partial seizures due to an underlying temporal lobe abnormality. MRI sequences used included $\mathrm{T} 1$ and $\mathrm{T} 2$ imaging, $\mathrm{T} 2$ mapping, ${ }^{1}$ IR, FLAIR, MPRAGE, and volumetric analysis of hippocampal volume. ${ }^{2}$ Preoperative MRI indicated hippocampal sclerosis (HS) in 45 cases and mass lesions in 29. All patients in this group underwent temporal lobectomy. HS was confirmed in 47 cases and mass lesions of variable histology in all 29 cases. Seventeen patients, all of whom showed gross anatomical abnormality on MRI, underwent hemispherectomy. Eight patients underwent craniotomy for extratemporal lesions (six stereotactically) and one had a stereotactic biopsy. Foreign tissue was detected in all pathological specimens.

Conclusion-MRI provides an accurate, reliable, and non-invasive method of localising structural abnormality and determining the underlying pathology in patients with medically intractable epilepsy. Advanced MRI techniques, however, are often required.

1 Jackson GD, et al. Neurology 1993;43:1793-9. 2 Cook MJ. Brain 1992;115:1001-15.

FRAMELESS STEREOTACTIC SURGERY FOR NEOCORTICAL LESIONAL EPILEPSY

PK Doshi, DGT Thomas, DR Fish, SD Shorvon. The National Hospital for Neurology and Neurosurgery, London, UK

The outcome of any lesional epilepsy depends on its complete surgical excision. Frameless stereotactic techniques not only help in delineating the lesion but, by their interactive information, also assist in complete excision. Eight patients with medically intractable epilepsy underwent frameless stereotactic guided excision. There were four men and four women, age range 21-36 (median 31) years. All patients were subjected to minimal craniotomies and complete excision of the lesion. The overall accuracy in locating the lesion was 1.26 (SD 0.35 ) $\mathrm{mm}$. They were evaluated at two months after surgery by MRI to confirm the extent of resection, and for change in their seizure frequency. Seven patients had complete excision of their lesions. Six patients had not experienced any seizures after discharge (Engel grade I) and two patients had more than significant reduction (Engel grade II) in their seizures. Thus frameless stereotaxy is a valuable adjunct to open surgery for accurately localising and resecting epileptogenic lesions.

REVIEW OF THALAMOTOMY: THE

MANCHESTER EXPERIENCE

S Chatterjee, PL Richardson. Manchester Royal Infirmary, Manchester, UK

A review of 34 thalamotomies between 1984 and 1993 is presented. Twenty two procedures were performed for multiple sclerosis related abnormal movements, the remainder for other indications.

Preoperative tremor was scored with respect to each of rest, action, and terminal accentuation. Preoperative disability was scored using the method described by Fahn, et al. ${ }^{1}$ Similar scores were obtained at six months follow up and at yearly follow ups. The period of follow up varied from 10 months to 108 months (median 37 months). The thalamotomy was performed by the same surgeon using the Leksell frame and ventriculography for localisation.

Patients with multiple sclerosis were graded into early, established, or advanced categories. The results for those with early or established disease were favourable initially in 11 out of 13 procedures (83\%) although there was recurrence of tremor within 18 months in 10 of these $11(91 \%)$. The period of improvement varied from five to 18 months. In patients without multiple sclerosis, the results were more satisfying with improvement initially in $11 / 12$ procedures which was sustained at 18 months follow up in 8/11 cases.

We are convinced that there is a definite role for thalamotomy in patients with involuntary movements even if these are associated with demyelinating disease.

1 Fahn S, Tolosa E, Marin C. Clinical rating scale for tremor. In: Janovic J, Tolosa F, eds. Parkinson's disease and movement disorders. Baltimore: Urban and Schwarzenberg, 1988:225-34.

STEREOTACTIC LIMBIC LEUCOTOMY: A CONTINUING TECHNIOUE FOR THE TREATMENT OF AFFECTIVE DISORDERS ND Kitchen, HT Marsh, AE Richardson. Atkinson Morley's Hospital, London, UK

In 1973 Richardson and colleagues described the procedure of stereotactic limbic leucotomy. ${ }^{1}$ The technique is still in use at Atkinson Morley's Hospital and consists of the production of 14 lesions bilaterally in the cingulum bundle and the ventromedial quadrant of the frontal lobes.

After application of the Leksell frame under general anaesthetic a sitting LP is performed and air ventriculography and teleradiology used to calculate target sites. A thermistor electrode is then used to create electrocoagulative lesions of about $1 \mathrm{~cm}$ in diameter. Electrophysiological corroboration of electrode placement is achieved by monitoring the effects on breathing of stimulation and lesioning. Postoperative MRI is used to assess the site and size of lesions.

Since 1987 stereotactic limbic leucotomy has been successfully performed on 23 patients with severe refractory affective disorders (depression (five), Gilles de la Tourette syndrome (two), and obsessivecompulsive disorders (16)). Neurological complications have been minor - namely, transient incontinence (five), confusion (one), and lethargy (four) and one temporary facial palsy.

The current technique of stereotactic limbic leucotomy is simple, safe, and accurate. It retains its place in the treatment of those few patients with life threatening affective disorders refractory to other therapeutic modalities.

1 Kelly D, Richardson AE, Mitchell-Heggs N. Br F Psychiatry 1973;123:133-40.

PREOPERATIVE DIAGNOSIS OF NEUROVASCULAR COMPRESSION OF THE TRIGEMINAL NERVE USING MAGNETIC RESONANCE

TOMOANGIOGRAPHY

LT Dunn, JF Meaney, PR Eldridge, JB Miles, TE Nixon. The Walton Centre for Neurology and Neurosurgery, Liverpool, UK

Microvascular decompression (MVD) is a well recognised treatment for trigeminal neuralgia (TGN). Hitherto, however, it has been impossible to demonstrate neurovascular compression (NVC) preoperatively.

The use of high definition magnetic resonance tomoangiography (MRTA) was investigated in the preoperative assessment of 52 patients with TGN (54 symptomatic and 50 asymptomatic nerves) and 25 healthy volunteers (50 nerves)

MRTA findings-(a) TGN: $90 \%$ (48/54) had NVC in the axilla, and a further $4 \%$ within $5 \mathrm{~mm}$ of this region. Only two patients showed no evidence of NVC (5\%). In $18 \%$ of cases NVC was thought to be venous and in $16 \%$ compression from more than one vessel was demonstrated. (b) Controls: NVC was present in the axilla of the nerve in only $8 \%$ of cases; in $9 \%$ there was vascular contact within $5 \mathrm{~mm}$ of this zone, and in a further $14 \%$ contact occurred more distally on the trigeminal nerve.

Surgical correlation-In all but one case surgical findings agreed with the preoperative MRTA findings. In this case NVC was predicted to be in the axilla but found some $5 \mathrm{~mm}$ away. In four early cases veins and not arteries were found at surgery. Retrospective analysis of the MRTA could demonstrate this distinction. Two cases had persistent pain after a technically satisfactory MVD. Postoperative MRTA showed a second vessel causing NVC. To date one of these cases has successfully undergone reexploration.

Conclusion-MRTA is a sensitive and specific method for demonstrating NVC. It is a useful aid to preoperative decision making and is of value in avoiding incomplete operative decompression. It is now a standard preoperative investigation of $\mathrm{TGN}$ at the Walton Centre. 
BRAIN STEM AUDITORY EVOKED POTENTIALS DURING CEREBELLOPONTINE ANGLE SURGERY

TRK Varma, A Forster. Dundee Royal Infirmary, Dundee, UK

Objective-To review and evaluate the use of brain stem auditory evoked potentials (BSAEPs) during cerebellopontine angle surgery.

Design-A retrospective view of 46 patients undergoing cerebellopontine angle surgery where BSAEP was monitored.

Patients-Thirty four patients undergoing microvascular decompression (MVD) had peroperative BSAEP monitoring in an attempt to reduce the incidence of hearing loss during this procedure. ${ }^{12}$ Nine patients with acoustic neuromas had BSAEP monitoring to assist in hearing preservation. Three patients underwent vestibular neurectomy for intractable Meniere's disease where BSAEP helped identify and preserve the auditory nerve.

Outcome measures-The value of BSAEP monitoring during cerebellopontine angle surgery in relation to the incidence of hearing loss.

Results-Peroperative changes in BSAEP were seen often during MVD procedures and these were readily reversed by reduction in retraction. Long term hearing loss was seen in only one patient where there were technical problems with the monitoring. BSAEP helped identify and preserve the auditory nerve in acoustic neuroma surgery. Hearing was preserved in six out of nine patients. BSAEP confirmed the identity and helped preserve function of the auditory nerve during vestibular neurectomy.

Conclusions-BSAEP monitoring during cerebellopontine angle surgery is a useful method of identifying the auditory nerve and helps prevent damage to the nerve.

1 Fritz W, Schafer J, Klein HJ. Hearing loss after microvascular decompression for trigeminal neuralgia. $\mathcal{f}$ Neurosurgery 1988; 69:367-70.

2 Friedman WA, Kaplan BJ, Gravenstein D, Rhoton AL. Intra-operative brain stem auditory evoked potentials during posterior fossa microvascular decompression. $\mathcal{f}$ Neurosurg 1985;62:552-7.

LONG TERM FACIAL NERVE FUNCTION AFTER ACOUSTIC NEUROMA SURGERY

BA Bell, NF Weir, KS O'Neill. Atkinson Morley's Hospital, London, UK

Objective-Permanent loss of facial nerve function is a significant cause of morbidity after successful surgery to remove large acoustic neuromas, and all of the existing techniques to improve a postoperative facial palsy have drawbacks. A glued sural nerve graft has been used when the facial nerve was divided in achieving total removal of large tumours and compared with the long term results from non-grafted patients.

Design-Ninety acoustic neuromas have been removed using intraoperative facial nerve monitoring over the past seven years, and in $69(77 \%)$ the facial nerve was preserved. In $21(23 \%)$ the facial nerve was divided in achieving total tumour removal, and in 16 of these patients a sural nerve graft was placed between the divided ends of the facial nerve, and fibrin tissue glue was used to secure the neural anastomosis. In five patients a direct end to end repair was possible without the need for a graft. Facial function was assessed using the facial paralysis prognostic index (FPPI), ${ }^{1}$ and the House and Brackmann grading system. ${ }^{2}$

Results - Two year follow up was available for three patients with a direct repair and eight patients with sural grafts. Good facial function (FPPI 5 to 8) was achieved in seven patients $(64 \%)$, satisfactory facial function (FPPI 4 ) in two (18\%), and poor function where graft failure was considered to have occurred (FPPI 0 to 3 ) in two patients $(18 \%)$. Of the 51 patients with intact facial nerves with two year follow up available, good facial function was achieved in $40(78 \%)$, satisfactory facial function in three $(6 \%)$, and poor function in eight patients $(16 \%)$.

Conclusion-Fibrin glue facial nerve grafting is simple to perform at the end of the operative removal of a large acoustic neuroma and restores satisfactory facial function at two years to $82 \%$ of patients. This is similar to the two year outcome for patients where the facial nerve was thought to be anatomically intact at the end of tumour removal.

1 Kerbavaz RJ, Hilsinger RL, Adour KK. The facial paralysis promostic index Otolaryngol facial paralysis prognostic index.

2 House JW, Brackmann DE. Facial nerve grading system. Otolaryngol Head Neck Surg 1985;93:146-7.

CHANGES IN PROTON MRS OF PERITUMOURAL OEDEMA BEFORE AND AFTER

DEXAMETHASONE

P Chumas, A Stewart, B Condon, D Oluoch-Olunya, D Hadley, G M Teasdale. Institute of Neurological Sciences, Glasgow, UK

Objective-To date the mechanism of action of dexamethasone in the treatment of brain tumours is poorly understood. We have utilised MRI and MR spectroscopy (MRS) to further elucidate potential modes of action.

Design-Proton MRS using a Siemens 1.5 $\mathrm{T}$ system pre and then 12 hours post administration of dexamethasone.

Subjects-Eight patients (three men, five women, mean age 54) with malignant gliomas were examined using MRI and MRS pre dexamethasone administration and then within 12 hours after administration and comparison made between ipsilateral and contralateral white matter.

Results-There was no significant change in the unsuppressed water peak in either of the regions following dexamethasone, which supports the findings of Bell, ${ }^{1}$ that peritumoural oedema takes days to alter. Regions over the oedema had significantly ( $p<$ 0.05 ) higher unsuppressed water than the contralateral regions. In all cases NAA/Cho and NAA/Cr decreased after the administration of dexamethasone. Lactate was found in the oedematous region of one patient predexamethasone and doubled postadministration. In two other patients measurable lactate peaks appeared post administration.

Conclusion-Increases in lactate may represent anaerobic glycolysis in oedematous and hypoxic white matter. ${ }^{2}$ Changes in NAA are difficult to interpret but may represent attempts at white matter repair.

1 Bell BA, Smith MA, Kean DM, et al. Brain water measured by magnetic resonance imaging: maging: Correlation with direct estimation and changes following mannit

2 Cruickshank GS, Rampling R. Acta Neurochir 1994;60(suppl):375-7.
INCIDENCE OF INTRACRANIAL TUMOURS IN LOTHIAN REGION

R Grant, D Collie. Western General Hospital, Edinburgh, UK

Objective-To identify the incidence of intracranial tumours in the Lothian Region.

Design-Audit of all incident cases of CT identified intracranial tumour in SE Scotland. Data were obtained from all CT cranial scans performed in SE Scotland (1989 and 1990), supplemented by neurology, neurosurgery, neuro-oncology, neuropathology, radiation therapy, and endocrine databases in Lothians and SMR6 data. Lothian region patients were identified by post code. Lothian region population was obtained from the April 1991 census.

Patients-A total of 578 patients with intracranial tumour of any type was analysed by tumour type (primary intracerebral, secondary intracerebral, benign intracranial extra-cerebral, others). Identification of pituitary microadenoma was based on hyperprolactinaemia with or without CT.

Outcome measures-Incidence of specific intracranial tumours in Lothian Region in cases/100 000 population/year.

Results-Incidence of all intracranial tumours of any type in the Lothian Region is $28 / 100000 /$ year. Primary intrinsic brain tumours had an incidence of nine (gliomas $7 \cdot 3)$. Incidence of secondary intracerebral tumour was $13 \cdot 1$, meningioma $2 \cdot 5$, pituitary tumours $2 \cdot 3$ (macroadenoma $1 \cdot 0$ ), acoustics 0.6 , and others 0.6 .

Conclusion-Cancer registration data in Scotland, and single institution studies have significantly underestimated the incidence of brain tumours.

THALLIUM AND HMPAO SPECT EVALUATION OF MALIGNANT BRAIN TUMOURS: EXTENT OF SURGICAL RESECTION, TUMOUR REGROWTH, AND RESPONSE TO TREATMENT

GS Cruickshank, J Patterson, D Hadley. Institute of Neurological Sciences, Glasgow, UK

Objectives-Postsurgical, residual viable tumour volume and recurrence during or after therapy has been followed by SPECT studies to determine suitability for determining surgical prognosis, tumour recurrence, radiation necrosis, and malignant change in tumours.

Design and patients - Thirty patients with brain tumours had preoperative ${ }^{201} \mathrm{~T} 1$ and HMPAO SPECT scans, again within five days of operation, at six weeks, and at 12 weeks with further scans as appropriate. Tumour and contralateral brain indices were evaluated together with estimates of volume of uptake by tumour.

Outcome measures-(a) Total volume $\left(\mathrm{cm}^{3}\right) ;(b)$ tumour to brain ratio; (c) coregistration CT/MRI/SPECT.

Results-The most useful finding was the ability to detect tumour recurrence at a much earlier stage than either CT or MRI. The most viable portions of tumour could be selected for biopsy or removal and these did not always coincide with the morphological picture presented by conventional scanning. The response to therapy could be followed.

Conclusions ${ }^{-201} \mathrm{~T} 1$ is a useful technique for following tumour regrowth and response to treatment. It can assist in selecting the areas to biopsy tumours and can provide a map of 
viable tumour location to use in conjunction with MRI or CT operative planning. This type of planning offers the possibility of selecting patients for early aggressive treatment of their tumours that have relapsed or show malignant change before neurological damage has progressed.

ENDOSCOPIC TREATMENT OF COLLOID CYSTS $P$ Barlow. Institute of Neurological Sciences, Glasgow, UK

Six patients with a colloid cyst of the third ventricle underwent endoscopic drainage, or attempted drainage, of the cyst. In five patients the procedure was successful in that it produced relief of symptoms with no serious complications. In one patient the cyst, an incidental CT finding, could not be found.

The technique is described and the advantages and disadvantages discussed. The main disadvantage of endoscopic drainage is that complete excision of the cyst does not seem feasible; therefore, because the risk of recollection is unknown, long term follow up will be required.

Open surgical excision is probably still the procedure of choice in most patients.

EFFICACY OF OPERATION FOR

MICROPROLACTINOMA: SUSTAINED

REMISSION AT 10 YEARS

GM Teasdale, JA Thomson, DA Davies, $H$

McLaren. Institute of Neurological

Sciences, Glasgow, UK

Objectives-To define the long term (10 years) results of operation for a microprolactinoma.

Design-Case record and postal follow up of 61 patients previously reported, ${ }^{1} 75 \%$ of whom had a normal prolactin (PRL) after five years.

Subjects-Forty five patients who were traced at 10 years.

Outcome measures-Serum PRL $<360$ mU/l.

Results-Thirty three patients (74\%) had a normal serum PRL. Relief of symptoms was noted in $76 \%$ of those with amenorrheoa, $87 \%$ with galactorrhoea, and $83 \%$ with infertility.

Conclusions-The results contradict a notorious previous report of recurrence of hyperprolactinaemia in $>50 \%$ of patients. ${ }^{2}$ Selective microadenomectomy is a useful option in management of a microprolactinoma.

1 Thomson JA, Teasdale GM, Gordon D, et al. Treatment of presumed prolactinoma by transphenoidal operation: early and late results. $B M \mathcal{F}$ 1985;291:1500-3.

2 Serri O, Raslo E, Beauregard $\mathrm{H}$, et al. Recurrence of hyperprolactinaemia after selective transphenoidal adenomectomy in women with prolactinoma. $N$ Engl $\mathcal{f} \mathrm{Med}$ 1983;309:280-2.

CLINICAL OUTCOME AND HAEMODYNAMIC TYPE OF CEREBRAL ARTERIOVENOUS MALFORMATIONS TREATED WITH LINAC RADIOSURGERY

AH Huneidi, DS-Montefiore, D Doughty, PN Plowman, F Afshar, KE Britton. St Bartholomew's Hospital, London, UK

Objective-To correlate the clinical outcome with the haemodynamic type of arteriovenous malformations (AVM) after LNNAC (linear accelerator) stereotactic radiosurgery (SRS).
Design-Comparison of angiographic obliteration rate and incidence of complications between fast and slow flow AVM after SRS.

Subjects-Eighty two patients (age 38 (12) years) with cerebral AVMs were treated with angiographic SRS (LNNAC; 5-Arc System; $25 \mathrm{~Gy}$ to $90 \%$ isodose). All were assessed clinically and with cerebral blood flow (CBF) studies before SRS and at three to six month regular intervals thereafter. The mean follow up period was 36 (10) months.

Outcome measures-Size of treated AVMs, incidence of complications, rate of angiographic obliteration of AVM, and changes in cerebral reserve (RI) and steal (SI) indices.

Results-AVM diameter ranged from 8 to $55 \mathrm{~mm}$ (< $25 \mathrm{~mm}$ (71\%), 25-40 mm (24\%) and $>40 \mathrm{~mm}(5 \%))$. Total obliteration rate was $70 \%$ at 24 months and $75 \%$ at 36 months. By contrast with slow flow AVMs for similar sizes, the fast flow AVM response to $S R S$ was quicker $(\Delta R I=0.24$ $(0.02) ; \Delta S I=-0.20(0.02)$ at 18 months; $\mathrm{p}<0.05)$. Three patients rebled at four, 18 , and 20 months; one of whom died $(50 \mathrm{~mm}$ diameter AVM). Six patients developed new neurological deficits; most were slow flow AVMs.

Conclusions-Fast flow AVMs show a quicker response to radiosurgery with a lower complication rate than slow flow AVMs.

GIANT INTRACRANIAL ANEURYSMS:

A REVIEW OF 65 CASES

VS Mehta. All India Institute of Medical Sciences, New Delhi, India

Giant intracranial aneurysms defined as being larger than $2.5 \mathrm{~cm}$ in diameter are reportedly uncommon. Among 374 patients with intracranial aneurysms managed between January 1986 and September 1993, $65(17 \cdot 4 \%)$ patients had giant aneurysms. Among them 39 (60\%) presented with subarachnoid haemorrhage and 26 $(40 \%)$ as a mass lesion. In two patients the presentation was unusual.

In 58 patients $(89 \%)$ the aneurysm was in the anterior circulation (ICA 39, ACOM 11, and MCA eight), in seven patients it was in the posterior circulation (BA four and VA three) and there were multiple aneurysms in five patients. Nine patients developed partial or complete thrombosis of the aneurysm either spontaneously or iatrogenically (possibly the result of earlier percutaneous carotid angiography). In six patients surgery was not carried out because of a medical contraindication or poor clinical grading. Three patients refused surgery and one died before surgical intervention.

Fifty two surgical procedures were carried out on 45 patients. In 23 , the aneurysm was clipped, in 17 a carotid occlusion (immediate or gradual), and in two patients a trap ligation was performed. Four patients underwent a STA-MCA anastomosis before carotid ligation. In five patients a variety of methods were used and the procedure was abandoned in three patients.

HOW GOOD IS GOOD ON THE GLASGOW OUTCOME SCALE AFTER SUBARACHNOID HAEMORRHAGE?

TJ Pigott, M Deane. The Walton Centre for Neurology and Neurosurgery, Liverpool, UK
After subarachnoid haemorrhage (SAH) the patients in the good outcome group on the Glasgow outcome scale (GOS) do have some continuing problems. SF-36 is a newly validated patient centred assessment proforma developed to survey health status. ${ }^{1}$ The form has eight different areas covering physical, psychological, and social aspects.

The results of a pilot study are reported using the SF-36 assessment in patients who have been successfully treated for SAH with a good outcome on the GOS. A total of 29 patients were assessed using the SF-36. Responses were received from 26 (93\%). The age range was from 33-72 years; the male:female ratio was $1: 2$.

In each aspect of SF-36 there was a reduction in score compared with normative data. ${ }^{2}$ This was most pronounced in the areas of physical and mental function. Although these were worse than the normative data, they indicate less disruption to a normal lifestyle than in some other chronic conditions such as low back pain and menorrhagia, but not varicose veins.

1 Ware J, Sherbourne C. The MOS 36-item short form health survey (SF-36). Med Care short form health

2 Garratt A, Ruta D, Abdalla M, et al. The SF36 health survey questionnaire. BMF 1993; 306:1440-4.

REVERSED DRAINAGE OF BRAIN INTERSTTTIAL FLUID THROUGH VENULAR BLOOD-BRAIN BARRIER

V Kunanandam. Hull Royal Infirmary, Hull, UK

Objective-To assess brain venules as an additional pathway for brain interstitial fluid (ISF) drainage, ${ }^{1}$ particularly when there is a rise in brain tissue pressure (BTP).

Design-Electron microscope (EM) study of passage of intraventricular horseradish peroxidase (HRP) through ISF pathways during periods of normal and raised intracranial pressure (ICP).

Subjects-Ventricular perfusion with HRP was carried out in eight anaesthetised Wistar rats. The animals were killed by cardiac perfusion of fixative after exposure of the brain to a period of raised ICP. Eight control animals had no period of raised ICP.

Outcome measures-EM detected the position of HRP in the brain tissue and venules.

Results-HRP entry from the brain into venules as vesicles and through the tight junctions was observed in the experimental, but not the control animals.

Conclusions-Brain venules allow ISF drainage when the BTP rises: thus venules probably regulate BTP. The venular ISF drainage mechanism has important therapeutic implications in the management of fluid balance and ventilation in raised ICP states.

1 Cserr HF, Patlak CS. Secretion and bulk flow of interstitial fluid. In: Bradbury MWB, ed. of interstitial fluid. In: Bradbury MWB, ed. Physiology and pharmacology of the blood-brain
barrier. Berlin: Springer-Verlag, 1992: 245-61.

A STUDY OF PERIOPERATIVE LUMBAR CSF PRESSURE IN PATIENTS UNDERGOING ACOUSTIC NEUROMA SURGERY

R Laing, P Smielewski, M Czosnyka, D Hardy, J D Pickard, P J Kirkpatrick. Addenbrooke's Hospital, Cambridge, UK 
Objective-To measure changes in CSF pressure after acoustic neuroma surgery and to relate these to changes in cerebral blood flow velocity.

Design-Lumbar catheters were used to measure CSF pressure and transcranial Doppler (TCD) to measure flow velocity in the middle cerebral artery (MCA). Although lumbar catheters were sited no CSF was drained.

Subjects-Twenty five patients undergoing translabyrinthine or retromastoid excision of acoustic neuroma. All had preoperative MRI and histological confirmation after uneventful tumour excision.

Measures-ICP, amplitude of CSF pressure pulse wave, arterial blood pressure, and the correlation coefficient between amplitude of pulse wave and CSF mean pressure (RAP), which reflects exhaustion of compensatory reserve, were recorded every minute by a microcomputer; TCD flow velocity was recorded on a daily basis.

Results-All patients showed a statistically significant rise in CSF pressure and RAP (suggesting decreased cerebrospinal compensatory reserve) after surgery. These increases tailed off over the next four days. Changes in MCA flow velocity were usually independent of changes in ICP although in a few cases a significant positive correlation was found.

Conclusion-After acoustic neuroma surgery there is a transient disturbance of CSF dynamics with raised ICP and decreased compliance. The change in compliance is not consistently the result of increased flow velocity and possibly of cerebral blood volume. These findings may account for the significant reported incidence of postoperative CSF leakage.

NEUROSURGERY AND GERONTOLOGY: FACTS AND FIGURES

DP O'Brien, J Nagaria, D Rawluk. Beaumont Hospital, Dublin, Ireland

Objective-Neurosurgical experience of health care management for the geriatric population was reviewed, in particular the types of clinical presentations, neuropathology, management, and final functional outcome on discharge.

Design-Retrospective analysis of all admissions to the department of patients of 65 years and over, for a 12 month period.

Patients-One hundred and eighty patients were studied ( 80 men, 100 women) which accounted for $9 \%$ of all admissions that year.

Outcome measures-Clinical presentations, Glasgow coma score, CT findings, clinical course, management, and Glasgow outcome score on discharge.

Results-The age range was 65-91 (mean 72.3) years (median 72 years). Interhospital transfers $(n=137)$ accounted for $76 \%$ of admissions and were deemed "emergency" $(118 / 137=86 \%)$ or "urgent" $(19 / 137=$ $14 \%)$. The commonest presentations were headache $(29 \%)$, confusion $(22 \%)$, and hemiparesis (14\%). According to the Glasgow coma score (GCS), $8 \%, 16 \%$, and $76 \%$ had severe, moderate, and minor impairment of conscious level respectively. Eleven patients $(6 \%)$ were transferred intubated and ventilated. CT was performed in 151 patients $(84 \%)$ in whom tumour (21\%), chronic subdural haematoma $(15 \%)$, subarachnoid haemorrhage $(15 \%)$, and acute subdural haematoma (9\%) were diagnosed. Fifty patients (28\%) either did not require operative intervention or were unfit for surgery. Overall, intracranial tumours, cerebral trauma, intracranial haemorrhage, and spinal disease accounted for $28 \%, 22 \%, 18 \%$, and $10 \%$ of the geriatric admissions respectively. There were 14 deaths (8\%). On discharge, $33 \%, 21 \%$, $35 \%$, and $3 \%$ were categorised as full recovery, moderate disability, severe disability, and persistent vegetative states respectively. The overall hospital stay ranged from 1-57 days $($ mean $=12.7$ days; median $=10$ days).

Conclusions-Elderly patients constitute a major proportion of the neurosurgical workload. This has major medical, rehabilitation, and socioeconomic implications.

MR NEUROGRAPHY OF CERVICAL ROOTS AND BRACHIAL PLEXUS

AG Filler, FA Howe, CE Hayes, JR Griffiths, BA Bell, JS Tsuruda. Atkinson Morley's Hospital, London, UK, CRC Magnetic Resonance Research Group, London, UK, and University of Washington, Seattle, USA

Objectives-Nerve pathology involving the distal cervical roots and brachial plexus has been difficult to diagnose accurately. Precise placement of electrodiagnostic electrodes has been problematic and most imaging modalities have yielded poor results. The initial reports of magnetic resonance neurography all focused on simple linear nerves such as the sciatic nerve in the thigh and the median nerve in the upper arm. The technical problems involved in adapting MR neurography for use in the lower neck and proximal shoulder region were the subject of this investigation.

Design-Specialised radiofrequency coil arrays have been developed to provide good saturation of the brachial plexus and distal cervical roots. New pulse sequences have been implemented for use on a GE Signa $1.5 \mathrm{~T}$ clinical imager that allows the use of diffusion weighting as well as previously reported aspects of the technique. ${ }^{1}$

Subjects-There were two normal subjects and 18 patients with a variety of diseases.

Outcome measures-This approach has led to very effective images of the brachial plexus and distal cervical roots based on image conspicuity.

Results-Even mild radiculopathy may be associated with image changes in individual nerve roots which are detectable by this technique, whereas more severe injury also causes gross enlargement of the roots. In many cases these results have aided surgical planning.

Conclusions-Brachial plexus neurography has now proved technically feasible.

1 Filler AG, Howe FA, Hayes CE, et al. Magnetic resonance neurography. Lancet 1993;341:659-61.

MRI SIGNAL CHANGES IN DENERVATED MUSCLF

GA West, M Kliot. Atkinson Morley's Hospital, London, UK and University of Washington, Seattle, USA

Objectives-To evaluate peripheral nerve injuries using MRI in comparison with standard electrodiagnostic studies and clinical examination.

Design-All patients had sequential neurological examinations, EMG, and MR imaging. T1, T2, and short-tau inversion recovery (STIR) images were obtained with a $1.5 \mathrm{~T}$ magnet.

Patients-A total of 32 patients (10 females, 22 males, ages 4-72 years) with a wide variety of peripheral nerve lesions were evaluated.

Outcome measures-Serial clinical examinations, EMG, and STIR images were used to assess peripheral nerve function.

Results-A total of 52 MR studies was performed on 32 patients. The average time between onset of peripheral nerve symptoms and MRI in 16 patients was 176 (93) days. Increased STIR signal in muscle, as early as four days after onset of symptoms, was seen in cases of severe axonotmetic injuries (transection of axons producing severe denervation) and correlated with the degree of denervation. In cases of neuropraxic nerve injuries (conduction block without axonal loss) the STIR signal in muscle was normal.

Conclusions-Denervation changes in muscle can be seen using STIR imaging and correlate with EMG changes and clinical examination. The STIR images provide a panoramic visual representation of denervated muscles useful in localising and grading the severity of peripheral nerve injury secondary to disease or trauma. Thus STIR imaging may play an important part in prediction of outcome and formulation of treatment soon after peripheral nerve injury.

IMAGE DIRECTED SURGERY OF THE SPINE: PRELIMINARY EXPERIENCE WITH THE ISG VIEWING WAND

N Patel, I Nelson, B Cummins, D Sandeman. Frenchay Hospital, Bristol, UK

Interactive image guided surgery is applicable to a complete cross section of intracranial problems. Stereotactic techniques have not, however, been applied outside the cranium. The versatility of the ISG viewing wand has made it possible to make use of the system in the spine. Three spinal operations out of a total of 260 cases are reported. Two of these were anterior approaches to odontoid peg and skull base causing medullary compression. The third was a case of a small sacral osteoblastoma, producing lumbar back pain. Both cases of cervicomedullary compression had complex atlantoaxial disease with a major element of basilar invagination. Wand registration was carried out using the same technique described for intracranial procedures. The wand was used to plan the choice of approach, transoral or transmaxillary, to determine precisely the midline, and to determine the depth and extent of resection in drilling out the odontoid peg. In both cases registration accuracy was comparable with that obtained with intracranial procedures.

Registration to the rest of the spine cannot be carried out using the same techniques as used intracranially because of the lack of rigid fixation of the wand base to the patient. A method has been devised for overcoming this difficulty. Subjective assessment suggested that the accuracy of 
the registration was not as good as could be achieved intracranially and was of the order of $\pm 5 \mathrm{~mm}$. If the technique of registration can be perfected then this has other applications for spinal surgery-for example, the positioning of interpedicular screws and orienting unfamiliar approaches to the cervical spine. The system also has application in the surgery of pelvic disorders, such as fractures and tumours.

OPERATIVE TREATMENT OF THE TETHERED CORD SYNDROME: EVALUATION AND EARLY MORBIDITY

RW Kirollos, PT van Hille. General Infirmary, Leeds, UK

Objective-As the indications for surgical treatment of the tethered cord syndrome are increasing, it is essential to assess the surgical risks especially if surgery is to be undertaken as a prophylactic procedure.

Patients-Between June 1991 and May 1994, 20 patients underwent surgical treatment for the tethered cord syndrome. Their ages ranged from five months to 53 years. There were 14 women and six men. Twenty one operations were performed, 14 for progressive neurological deficit, three for progressive lower limb deformity, and four as a prophylactic procedure.

All patients underwent MRI to confirm the diagnosis. Cord tethering was due to a spinal lipoma in 12 cases, diastematomyelia in four, and a tight filum terminale or intradural adhesions in the remainder. Nine patients had undergone previous surgery to close a myelomeningocoele shortly after birth. Previous surgery did not affect the rate of subsequent successful untethering.

Results-There were four early postoperative complications; three CSF leaks, all requiring re-operation, and one patient showed an increase in established neurolog- ical deficit. None had new irreversible neurological deficit as a result of surgery.

Conclusion-This low rate of complications and the success of satisfactory untethering justifies surgery to prevent further neurological deficit as a prophylactic measure.

OUTCOME OF POST-TRAUMATIC

SYRINGOMYELIA

S Sgouros, B Williams. Midland Centre for Neurosurgery and Neurology, Smethwick, UK

Traumatic paraplegia is the commonest cause of non-hindbrain related syringomyelia. Forty three patients with a mean age of 33.7 years at presentation have been treated at the Midland Centre between 1972 and 1992. A variety of treatment strategies have been employed over the years, including syringosubarachnoid and syringopleural shunts, cord transection, pedicled omental graft transposition and recently wide decompressive laminectomy with formation of a surgical meningocele.

The overall postoperative complication rate was $10.6 \%$. Problems specific to the operation type included dislodged and blocked drains (six). Acute gastric dilatation was seen after pedicled omental graft (one). One drain became infected. At 10 years only $60 \%$ of the drains inserted remained functioning. A higher than expected rate of cervical spondylotic myelopathy has been noted. Two patients developed Charcot's joints.

Twenty two patients were asked to score themselves with regard to limb function and performance of daily living activities and $32 \%$ reported improvement.

Since the use of MRI became widespread, it has become apparent that decompressive laminectomy with opening of the subarachnoid spaces is particularly effective in controlling the syrinx cavity. In complete paraplegics, cord transection is an effective alternative. Pedicled omental grafting was associated with poor outcome and increased complication rate and has been abandoned.

SYRINGOMYELIA: A REVIEW OF 51 PATIENTS SY Sharif, MS Eljamel, CN Pidgeon. Beaumont Hospital, Dublin, Ireland

Syringomyelia is a chronic progressive disease with current management being controversial. We reviewed 51 consecutive patients (24 men and 26 women) who were treated surgically. The mean age at presentation was 40.7 years. Main presenting symptoms were cervical pain, sensory disturbance, and motor deficit. Significant tonsillar herniation was present in $90 \%$ of patients, $18 \%$ had hydrocephalus, and $12 \%$ had spina bifida. Average duration of observation between diagnosis and surgical intervention was one year. Adequate documentation of clinical course before surgery was available in 39 patients, and of these $82 \%$ reported deterioration. Half of these patients had repeat MRI and $78 \%$ showed progression of their syrinx. Ventricular shunts were inserted in nine patients, posterior fossa decompression was performed in 40 patients, and syrinx drainage in 10 patients. There were no deaths, $70 \%$ of patients complained of severe headache, $4 \%$ had a wound infection, and $2 \%$ had a temporary CSF leak. Adequate postoperative follow up was available in $\mathbf{4 2}$ patients, of whom $55 \%$ improved and $16 \%$ progressed. Postoperatively, MRI was performed on 15 patients, and eight showed collapse of syrinx and six remained unchanged. Thus shunting the hydrocephalus is advocated as the first line of surgical treatment, foramen magnum decompression for Arnold-Chiari malformation, and lastly syringotomy, if required. 